\title{
An empirical study on the coordinated economic development of Shen-Guan-Hui economic circle
}

\author{
Chunshang $\mathrm{Wu}^{1,2}$ \\ ${ }^{1}$ Heyuan Polytechnic, Heyuan city, Guangdong Province, 517000,China \\ ${ }^{2}$ City University of Macau, Macau, ,China \\ wuchunshang1023@163.com
}

Keywords: Shen-Guan-Hui Economic Circle; Economic coordinated development; Coordination degree.

\begin{abstract}
The uncoordinated regional development has become the soft spots of Guangdong's economy. Shen-Guan-Hui Economic Circle was put forward to promote the development of Heyuan and Shanwei, in order to promote the coordinated development of regional economy. Through building regional economic coordinated development measurement model, we make an empirical analysis on the degree of coordinated development of Shen-Guan-Hui Economic Circle. At last we put forward we put forward some suggestions for the coordinated development of Shen-Guan-Hui Economic Circle.
\end{abstract}

\section{Introduction}

Since the 1990s, the coordinated development of regional economy has become a hot research topic in China. It is also a key consideration when the government equationtes policies. The research on the coordinated development of regional economy mainly focuses on two aspects. (1) Research on the connotation and Realization of regional coordinated development. In the 1990s, Qinghai Jiang summed up the contents of regional coordinated development and put forward seven indicators to judge the coordinated development of economy, such as self-development ability, regional disparity, regional development advantage, flow of production factors, regional economic connection, unified market and development of regional productive forces ${ }^{[1][2]}$. Dadao Lu (1997) had constructed a "four in one " mechanism of regional coordinated development ${ }^{[3]}$. (2) Measure the degree of coordinated economic development, and test it empirically. Qinmei Wang (2007) holds that the coordinated development of regions should satisfy four conditions: narrowing the income gap between regions; jointly developing regions; paying attention to equity and promoting the realization of common prosperity under the Pareto improvement of regional interests ${ }^{[4]}$. Chenglin Qin (2011) studied the concept, judgment criteria and evaluation methods of regional economic coordinated development [5], and then verified the level of regional economic coordinated development in Guangdong [6].

Guangdong is the developed region in China. Guangdong GDP has been the first in past 29 years, but there is a big gap in Guangdong's internal economic development. The Pearl River Delta accounts for nearly $80 \%$ of the province's GDP. But Shanwei's GDP was only $0.9 \%$ of the province in year 2017. In order to solve the serious imbalance of economic development, Guangdong Province has issued a series of policies. In 2014, Heyuan and Shanwei were approved to join the Shen-Guan-Hui Economic Circle to promote coordinated economic development by building an integrated economic circle. After The Shen-Guan-Hui Economic Circle was put forward, the coordinated development of regional economy has been widened and narrowed compared with the previous economic development gap. There is still no research on this area.

\section{The model for measurement of the level of economic coordinated development}

The connotation of the coordinated development of regional economy has not yet formed a unified view. Some scholars believe that the coordinated development of regional economy means the 
coordinated development of regional economy with society, ecological environment and resources. Others believe that the coordinated development of regional economy means the coordinated development of industries within the region. But the mainstream view holds that the coordinated development of regional economy means the coordinated development of industries within the region. Economic coordinated development is to guide the rational division of labor and cooperation between regions, to achieve the sustainable development of the relevant regions, and the economic gap between them tends to narrow.

This paper References the definition of Chenglin Qin in 2011: the coordinated development of regional economy means that: Under the opening of regional economy, the economic links between regions are closer, the economic development is promoting, the regional economy is developing continuously and the economic differences tend to narrow ${ }^{[7]}$. And referring to its measurement method, the coordinated development level of regional economy is divided into three aspects: regional economic ties, regional economic growth, regional economic differences. Because these three are the important contents of regional economic coordinated development, we can get the index of regional economic coordinated development after weighting them.

\subsection{Regional economic linkage measurement}

Regional economic linkages refer to the existence of close economic ties and regional division of labor between regions. For example, the former store and factory model in Dongguan and Hong Kong have a close economic relationship between Hong Kong and Dongguan. Using the method of spatial econometrics, the Moran's I coefficient is used to measure regional economic relations. The equation is as shown in equation (1).

$$
I=\frac{n \sum_{i=1}^{n} \sum_{j=1}^{n} W_{i j}\left(x_{i}-\bar{x}\right)\left(x_{j}-\bar{x}\right)}{\sum_{i=1}^{n} \sum_{j=1}^{n} w_{i j} \sum_{i=1}^{n}\left(x_{i}-\bar{x}\right)^{2}}
$$

$\mathrm{n}$ is the number of regions, and the variables $\mathrm{X}_{\mathrm{i}}, \mathrm{X}_{\mathrm{j}}$ represent the per capita GDP of the region $\mathrm{i}$ and region $\mathrm{j}$, and $\mathrm{W}_{\mathrm{ij}}$ is the spatial neighboring weight matrix of the region $\mathrm{i}$ and region $\mathrm{j}$. The method of calculation is shown in equation (2).

$$
w_{i j}=\left\{\begin{array}{cc}
1 & \text { Refion } \mathrm{i} \text { and area } \mathrm{j} \text { are adjacent } \\
0 & \text { others }
\end{array}\right.
$$

Moran' $s$ I has a value between -1 and 1 . If Moran' $s \mathrm{I}>0$, it means that the region $\mathrm{i}$ is positively correlated with the economy of region $\mathrm{j}$, and the inter-regional economy is closely linked.The closer to 1 , the closer the economic relationship between adjacent regions is. On the other hand, if Moran ' $\mathrm{s}$ $\mathrm{I}<0$, it indicates that the economy of region $\mathrm{i}$ and region $\mathrm{j}$ is negatively correlated, and the regional economic relationship is weak.

\subsection{Regional economic growth state measure}

The state of regional economic growth refers to the rate of economic growth in various regions. The regional growth state is measured by the coefficient of variation of regional economic growth. The equation is given by equation (3).

$$
\alpha_{t}=\frac{\sqrt{\sum_{j}\left(y_{j}-\bar{y}\right)^{2} / n}}{\bar{y}}
$$

$\alpha_{t}$ refers to the economic growth state coefficient. $y_{j}$ is the regional $\mathrm{j}$ GDP growth rate, $\bar{y}$ is the average growth rate of GDP. The larger of $\alpha_{t}$, the greater the difference in regional economic growth, indicating that the positive effect of inter-regional economic growth is worse. 


\subsection{Regional economic difference state measurement}

Since per capita GDP can better reflect the economic strength of a region, the coefficient of variation of per capita GDP is used to measure the state of regional economic disparity. The regional economic disparity state coefficient is obviously smaller, indicating that the regional economic level is small and the degree of coordination is high. Its calculation equation is shown in equation (4).

$$
\beta_{t}=\frac{\sqrt{\sum_{j}\left(x_{j}-\bar{x}\right)^{2} / n}}{\bar{x}}
$$

\subsection{Regional economic coordination degree measurement}

Since regional economic linkages, regional economic growth states, and regional economic differences constitute three aspects of coordinated regional economic development, and are equally important, the three measurement indicators are given the same weight. The larger of Moran's I, the higher degree of coordinated development, However, The smaller the regional economic growth state and the regional economic difference state, the higher the degree of coordinated development.So 1-I is used. The formula for calculating the regional economic coordination coefficient is shown in equation (5).

$$
z_{t}=\frac{\left\{\left(1-I_{t}\right)+\alpha_{t}+\beta_{t}\right\}}{3}
$$

\section{The empirical analysis of regional coordinated development of Shen-Guan-Hui Economic Circle}

\subsection{Regional economic linkage measurement of Shen-Guan-Hui Economic Circle}

The data from year 2000 to 2017 are substituted in equation (1) and calculated by GeoDa software. The results are shown in Figure 1.

As can be seen from Figure 1, the economic links in Shen-Guan-Hui Economic Circle have been strengthened since 2000, but since 2008, the regional economic links have begun to weaken. That may be because after the 2008 financial crisis, in order to stabilize economic growth, cities had make industrial adjustments, and the economic links was weakened. After 2014, the economic links have been Strengthened because in 2014 Heyuan and Shanwei officially approved to join the Shen-Guan-Hui economic circle, local governments have taken a series of measures to promote regional economic integration.

\subsection{Regional economic growth state measure of Shen-Guan-Hui Economic Circle}

Substitute the data from year 2000 to 2017 in equation (3), we get the regional economic growth status of Shen-Guan-Hui economic circle, as shown in Figure 2.

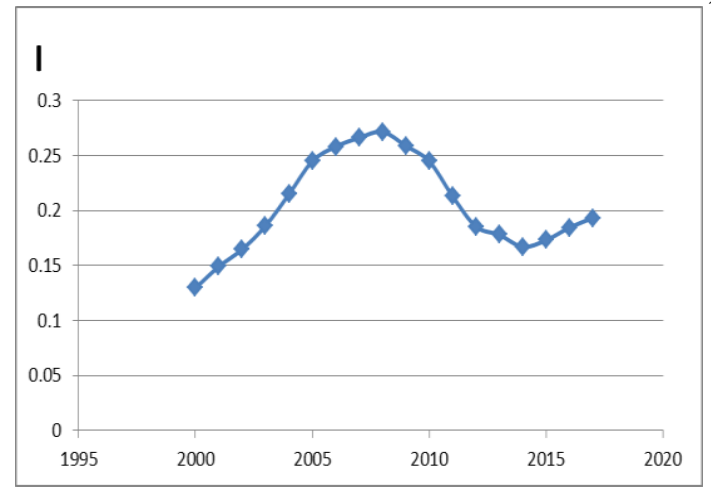

Fig. 1. Calculation results of Moran's I

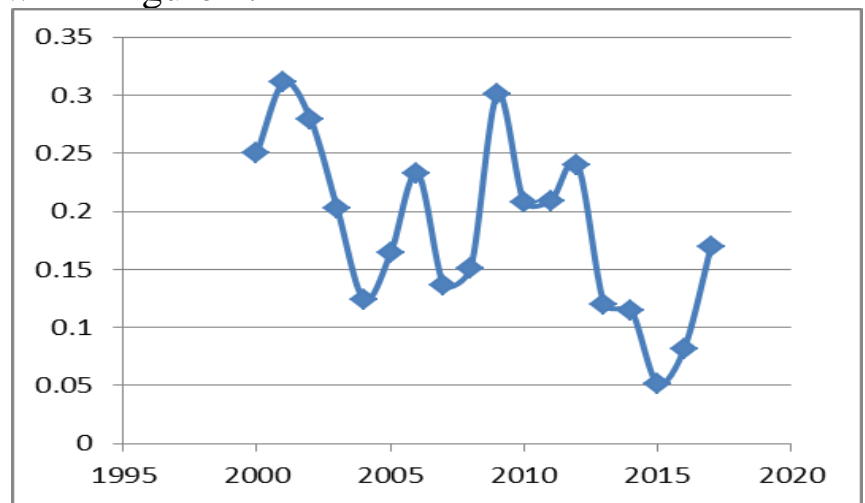

Fig. 2. State coefficient of regional economic growth 
As can be seen from Figure 2, the economic growth disparity of Shen-Guan-Hui economy changes greatly, but regional economic disparity has a narrowing trend. Before 2006, the difference of regional economic growth was mainly due to the lower economic growth rate in Heyuan and Shanwei, which further widened the economic gap between them and Shen-Guan-Hui. In 2009, the difference of regional economic growth is great, mainly because Dongguan GDP growth rate is only 5.3\%, because the impact of financial crisis. The difference coefficient of economic growth in 2017 was mainly due to the growth rate of GDP in Heyuan is only $5.1 \%$.

\subsection{Regional economic difference state measurement of Shen-Guan-Hui Economic Circle}

After substituting data in equation (4), the result is shown in Figure 3. From the figure 3, we can see that the economic disparity of Shen-Guan-Hui Economic Circle has been declining, especially after 2010 , the coefficient of economic disparity has been maintained at about 0.68. The economic disparity of Shen-Guan-Hui Economic Circle has been effectively controlled and has a tendency to reduce.

\subsection{Regional economic coordination degree measurement of Shen-Guan-Hui Economic Circle}

Substitute the data from first three steps in equation (5), then the results are shown in Figure 4. As can be seen from Figure 4, the overall regional economic coordination coefficient is declining, indicating that the Shen-Guan-Hui Economic Circl has a higher degree of economic development coordination, but in 2009 and 2017, the level of regional economic coordinated development was declined. In 2009 it was mainly affected by the world financial crisis, and 2017 was mainly affected by the slow growth of Heyuan.

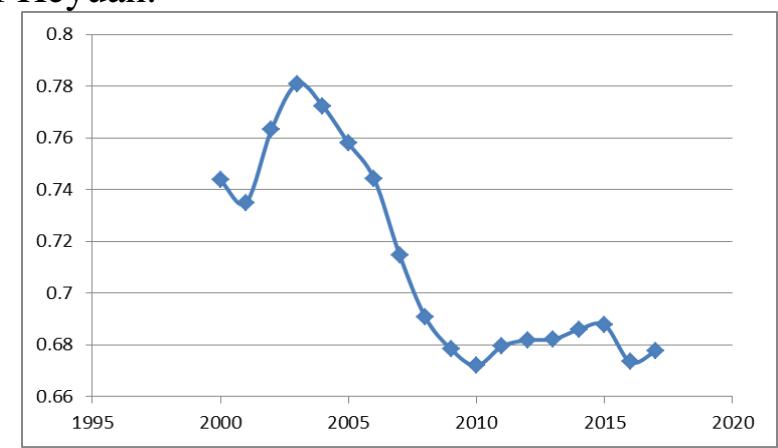

Fig. 3. State coefficient of regional economic disparity

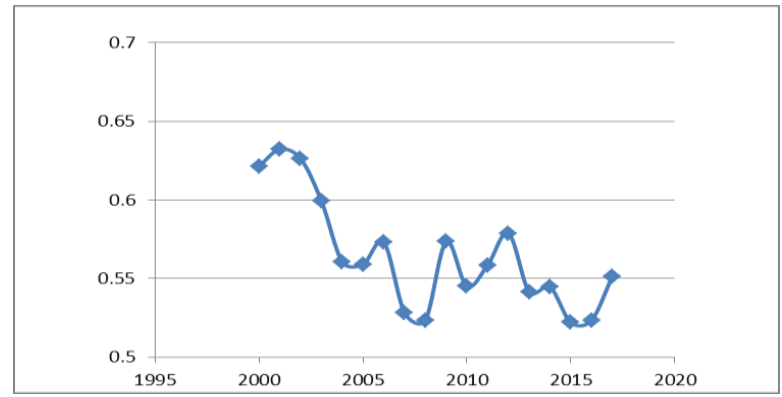

Fig.4. Regional economic coordination degree coefficient

\section{Summary}

Shen-Guan-Hui Economic Circle's regional economic coordinated development is mainly due to the strengthening of regional economic links, and the reduction of regional economic disparities.So enhance the level of coordinated development We must start from two aspects: strengthening regional economic ties and accelerating the rapid economic growth of Heyuan and Shanwei. Specific measures are as follows: (1) Strengthen the construction of regional infrastructure so that the economic circle can be interconnected. (2) We should rationally plan the industrial layout and functional zoning of the economic circle so as to achieve industrial complementarity and fruit sharing.(3) Through joint party and government meetings and other means, break regional barriers, promote the free flow of technology, and build a unified and open market environment. (4) In order to promote the rapid economic development of the two cities and narrow the regional economic gap, we should Increase industrial transfer and support to Heyuan and Shanwei.

\section{Acknowledgement}

This research was financially supported by the Guangdong Provincial Department of Education Research Project(2017GWTSCX044)and the Heyuan social science joint project (Grant NO. HYSK16Z01). 


\section{References}

[1] Qinghai Jiang, On the coordinated development of regional economy, Research on Development, vol.1 ,pp. 37-40,1993

[2] Qinghai Jiang, Some theoretical issues on the coordinated development of regional economy, Research on Financial and Economic Issues, vol.6, pp. 49-54, 1995.

[3] Dadao Lu, On the Areal Cooperation and the Coordination Development of Regional Economy, Areal Research and Development, vol.1, pp. 44-47, 1997.

[4] Qinmei Wang, A new interpretation of the connotation of regional coordinated development, Gansu Social Sciences, vol. 6, pp. 46-50, 2007.

[5] Chenglin Qin, Hua Zhang, Chao Mao, Coordinated development of Regional Economy: concept discrimination, judgement standard and evaluation method, Reform of Economic System, vol. 4, pp. 34-38, 2011.

[6] Chenglin Qin, Yunfeng Zheng,The coordinated development level and policy effect of Guangdong's regional economy, Research on Development, vol. 3, pp.10-13, 2012. 